Check for updates

Cite this: Chem. Sci., 2019, 10, 6821

๑ All publication charges for this article have been paid for by the Royal Society of Chemistry

\section{Boosting the circularly polarized luminescence of small organic molecules via multi-dimensional morphology control $\dagger$}

\author{
Kai Ma, ${ }^{\text {ab }}$ Wenjie Chen, ${ }^{\text {bcd }}$ Tifeng Jiao, (D) *a Xue Jin, ${ }^{\mathrm{b}}$ Yutao Sang, (DD ${ }^{\text {bd }}$ Dong Yang, \\ Jin Zhou, ${ }^{b}$ Minghua Liu (D)*bcd and Pengfei Duan (iD)*bd
}

Achieving a higher dissymmetry factor is a crucial issue in developing circularly polarized luminescence $(\mathrm{CPL})$ materials. Here, by tailoring the solvent composition and the morphology of the same chiral emissive small molecules ( $R$ - or S-SPAn), circularly polarized emission with a boosted dissymmetry factor (two orders) was realized. It was found that by regulating the water fraction in the mixed $\mathrm{THF} / \mathrm{H}_{2} \mathrm{O}$, we were able to achieve kinetic control over association of chiral emissive $R$ - or S-SPAn into various nanostructures with OD nanospheres, 2D nanoflakes and 3D stacked nanoflakes. These nanostructures are all CPL active. Remarkably, the dissymmetry factors of the nanostructures were significantly enhanced compared to those of the molecules and further boosted in different morphologies, from $\sim 10^{-4}$ (OD nanospheres) to $10^{-3}$ (2D flake) to $\sim 10^{-2}$ (3D nanoflakes). The enlarged $g_{\text {lum }}$ value could be assigned to a good packing induced strong luminescence of an excimer. This strategy provides an efficient way to fabricate higher dissymmetry factor CPL organic nanomaterials by only changing the supramolecular architectures while using the same chiral small molecules.
Received 1st April 2019

Accepted 1st June 2019

DOI: $10.1039 / c 9 s c 01577 a$

rsc.li/chemical-science

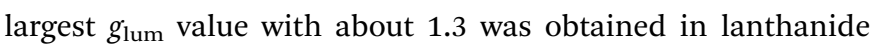
complexes, that is cesium tetrakis(3-heptafluoro-butylryl(+)-camphorato) Eu(III) complexes. ${ }^{24}$ On the other hand, in contrast to lanthanide complexes, research on simple organic molecules with CPL activity has become popular due to the inherent advantages of organic luminescent molecules. ${ }^{25,26}$ Compared with lanthanide complexes, the emission of pure organic CPL-active materials could be tuned more flexibly by regulating the electronic levels of the excited state. For instance, many strategies have been realized to modulate the electronic state of organic molecules, such as by chemical transformations of substituents, self-assembly and by changing the environment through external stimuli, e.g. temperature, $\mathrm{pH}$, concentration, light, magnetism and solvents. ${ }^{27-38}$ Unfortunately, the $g_{\text {lum value }}$ of organic systems is relatively low and generally falls in the range of $10^{-5}$ to $10^{-3}$, which precludes them from being the best candidates for CPL research. To amplify the luminescence dissymmetry factors, different approaches have been adopted, which include the formation of receptor-ion complexes, configurational changes upon binding with guest ions or molecules and self-assembly of chiral molecules. ${ }^{39-46}$ For example, Tang and co-workers, by introducing the concept of aggregation-induced emission, realized the amplification of $g_{\text {lum }}$, which could be regarded as an excellent approach for fabricating efficient CPL-active organic solid materials. ${ }^{25,47,48}$ Very recently, we have demonstrated that energy transfer, including Förster resonance energy transfer and the photon upconversion process, could remarkably amplify the $g_{\text {lum }}$ 


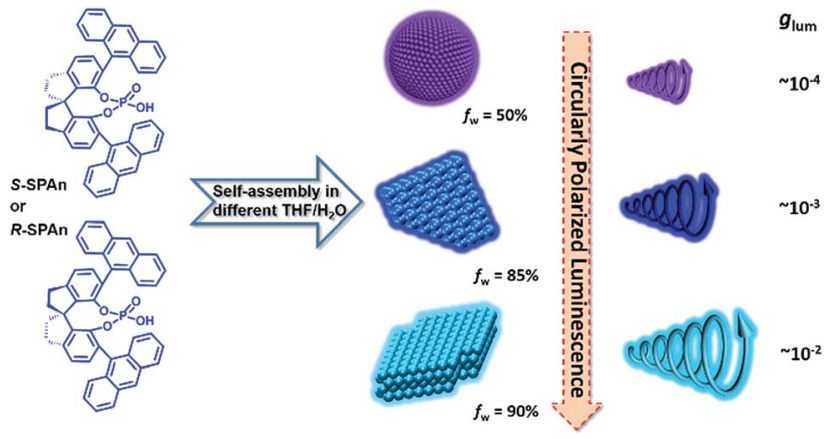

Scheme 1 Self-assembly of chiral molecules into different nanostructures in mixed $\mathrm{THF} / \mathrm{H}_{2} \mathrm{O}$ with different volume ratios. Upon increasing the volume fraction of water, assembled structures of nanoparticles, and 2D and 3D nanoflakes were obtained. The CPL of the aggregates obtained in various water fractions was gradually amplified and the $g_{\text {lum }}$ value of 3D nanoflakes finally increased by two orders of magnitude in comparison with the monomeric molecules.

value. ${ }^{49-51}$ Kawai and co-workers have found that self-assembly could be used as an approach for amplifying the $g_{\text {lum }}$ value. ${ }^{52}$ In addition, they have demonstrated that one-dimensional selfassembled nanostructures exhibited larger $g_{\text {lum values than }}$ zero-dimensional aggregates. However, detailed demonstrations of morphology-dependent circularly polarized emission with regulated large $g_{\text {lum }}$ values in pure organic molecule-based systems have rarely been reported.

Herein, we report an interesting CPL-active organic system, which shows morphologically dependent CPL activity with controllable $g_{\text {lum }}$ values, as shown in Scheme 1 . This category of molecules was originally synthesized as a privilege catalyst for asymmetric reactions. ${ }^{53,54}$ Different morphological features of the nanostructures from the same chiral small molecule could be flexibly tuned by changing the mixing ratio of THF and water. Upon increasing the volume fraction of water, the nanostructures changed from 0D nanospheres to 2D and 3D nanoflakes. By trapping the chiral molecule association into different supramolecular architectures, the $g_{\text {lum }}$ value of the specific nanostructures showed a remarkable amplification from $10^{-4}$ to $10^{-2}$. Particularly, compared with the monomeric state of the chiral emissive molecule, the $g_{\text {lum }}$ value showed an amplification factor of two orders of magnitude. Thus, avoiding tedious organic synthesis, by simply regulating the composition of solvents, there still is great potential for enhancing the CPL activity.

\section{Results and discussion}

Initially, the primary photophysical investigations of monomeric and aggregated $R^{-}$or $S$-SPAn were carried out by employing a series of optical testing methods. As shown in Fig. S1a, $\uparrow$ upon increasing the volume fraction of water, the emission colour varied from deep blue to cyan under a UV lamp. The emission spectra also showed an obvious red shift. The maximum emission peak of $R$-SPAn in pure THF solution was located at $422 \mathrm{~nm}$, which was identical to the one in the aggregate state in the mixed solvent $\left(\mathrm{THF} / \mathrm{H}_{2} \mathrm{O}=1 / 1\right)$ (Fig. 1a). Upon increasing the volume fraction of water $\left(f_{\mathrm{w}}\right)$ to $85 \%$, the emission spectrum showed a bathochromic shift to $432 \mathrm{~nm}$ with the emergence of a shoulder peak at $460 \mathrm{~nm}$. When the water fraction reached $90 \%$, the vibronic peaks tended to disappear and the maximum emission peak was located at $460 \mathrm{~nm}$. Interestingly, the emission quantum yield $\left(\Phi_{\mathrm{em}}\right)$ showed an increasing trend from 0.44 (pure THF solution) to 0.6 $\left(f_{\mathrm{w}} 50 \%\right)$. However, after increasing the water fraction to $85 \%$, the emission dramatically quenched $\left(\Phi_{\mathrm{em}}=0.04\right)$, which should be due to the aggregation-caused quenching of luminescence. Finally, upon increasing the water fraction to $90 \%$, the quantum yield reached 0.13 (Fig. S1b and S2a $\dagger$ ). As shown in Fig. S2b, $\dagger$ upon addition of water, the absorption spectra of $R$-SPAn showed a slight bathochromic shift and broadening, which indicated the formation of aggregates. The nature of different emitters of aggregates was investigated by emission lifetime measurement (Table S1 $\dagger$ ). The emission lifetime for $f_{\mathrm{w}} 0 \%$ and $50 \%$ exhibited a monoexponential decay with a lifetime of $8 \mathrm{~ns}$ for emission monitored at $422 \mathrm{~nm}$, which could be attributed to the luminescence of monomeric molecules. Upon increasing the water fraction to $85 \%$, the aggregates exhibited a fast biexponential decay and triple-exponential decay lifetime monitored at $432 \mathrm{~nm}$ and $460 \mathrm{~nm}$, respectively. The emergence of new emissive species with a lifetime of about $2.3 \mathrm{~ns}$ and a longer lifetime of about $6.8 \mathrm{~ns}$ may be attributed to the luminescence of the aggregates and excimer, respectively. The lifetime for $f_{\mathrm{w}}$ $90 \%$ monitored at $460 \mathrm{~nm}$ exhibited a triple-exponential decay and the emergence of a long-lived lifetime of about $10 \mathrm{~ns}$ could further confirm the formation of an excimer. ${ }^{55-57}$ It could be concluded that the formation of the aggregates and excimer was responsible for the characteristic spectral features observed at higher water fractions. ${ }^{58}$

To test the chirality of the monomeric and self-assembled states, circular dichroism (CD) and CPL measurements were carried out under different conditions. In pure THF solution, the CD spectra of the monomeric molecule showed an obvious Cotton effect corresponding to the absorption bands (Fig. 1b). The CD signals from 200 to $300 \mathrm{~nm}$ could be assigned to the electronic transitions of the spiral chromophore with axial chirality, ${ }^{59}$ while the signals in the range of 350-400 $\mathrm{nm}$ were assigned to the anthracene units, respectively. Mirror-image CD spectra were observed for the $R$ and $S$-enantiomers. Since the anthracene chromophores were achiral, the CD signals located at $350 \mathrm{~nm}$ to $400 \mathrm{~nm}$ could be assigned to the intramolecular chirality transfer from spiral chromophore to anthracene. The CD spectra of $R$ - or $S$-SPAn under various conditions are shown in Fig. S3. $\dagger$ The CD intensity corresponding to the anthracene chromophore exhibited amplification accompanying the increasing water fraction. We used the absorption dissymmetry factor $\left(g_{\mathrm{CD}}\right)$ to evaluate the change of chirality. The absorption of an asymmetric factor $\left(g_{\mathrm{CD}}\right)$ is defined as $g_{\mathrm{CD}}=\Delta \varepsilon / \varepsilon=2\left(\varepsilon_{\mathrm{L}}-\right.$ $\left.\varepsilon_{\mathrm{R}}\right) /\left(\varepsilon_{\mathrm{L}}+\varepsilon_{\mathrm{R}}\right)$, where $\varepsilon_{\mathrm{L}}$ and $\varepsilon_{\mathrm{R}}$ denote molar absorption coefficients of left and right circularly polarized light, respectively. The $g_{\mathrm{CD}}$ of the anthracene was amplified from $1.5 \times 10^{-4}(390$ $\mathrm{nm})$ to $3.1 \times 10^{-3}(412 \mathrm{~nm})$ (Fig. $\left.\mathrm{S} 4 \dagger\right)$. These results indicated that the stronger exciton coupling in well-ordered structures 
a)

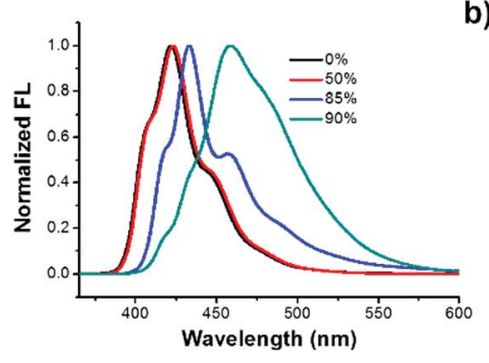

c)

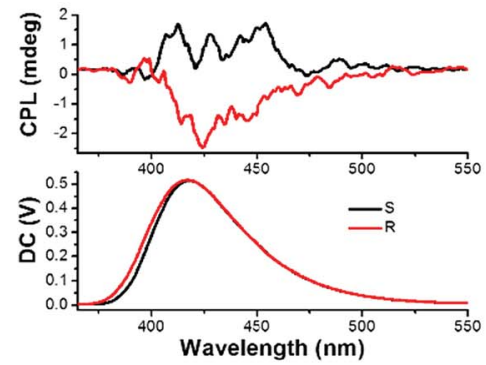

b)

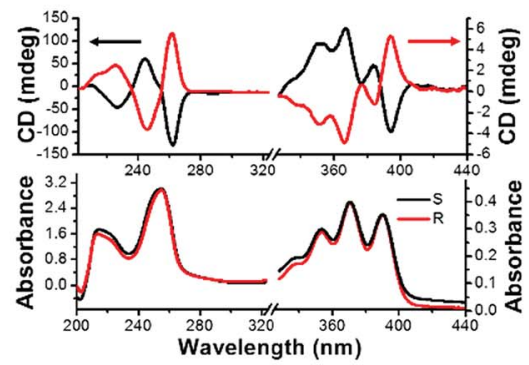

d)

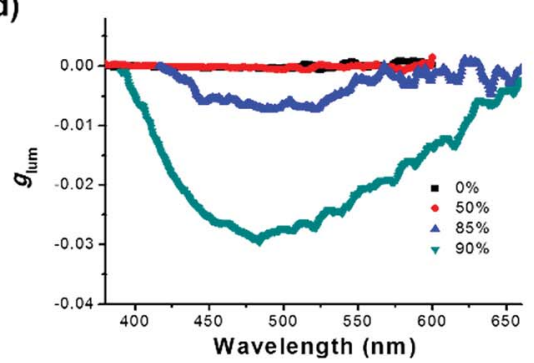

Fig. 1 (a) Fluorescence spectra of $R$-SPAn aggregates in various $f_{\mathrm{w}}\left(\lambda_{\mathrm{ex}}=320 \mathrm{~nm}\right.$; intensity normalized at a maximum value). (b) CD spectra of $R$ and S-SPAn in pure THF. (c) CPL spectra of $R$-/S-SPAn in pure THF. (d) Dissymmetry factor $g_{\text {lum }}$ of $R$-SPAn aggregates in various $f_{\mathrm{w}}$. Upon increasing the volume fraction of water, the maximum $g_{\text {lum }}$ for $f_{\mathrm{w}} 90 \%$ was amplified two orders of magnitude in comparison with the $g_{\text {lum }}$ for $f_{\mathrm{w}}$ $0 \%$ and $50 \%$. ([R-SPAn] $=1.5 \mathrm{mM}, \lambda_{\mathrm{ex}}=320 \mathrm{~nm}$ ).

could significantly amplify the $g_{\mathrm{CD}}$ by controlling the solvents. Thus, by controlling the morphology of nanostructures aggregated at various water fractions, amplified supramolecular chirality was obtained. The amplified $g_{\mathrm{CD}}$ laid the foundation for enhanced CPL as discussed below. The CPL spectra could provide more direct evidence for chiral dissymmetry in the fluorescence of the monomer and aggregates (Fig. 1c and S5†). Upon increasing the volume fraction of water, the intensity of the CPL was obviously enhanced. The extent of chiral dissymmetry in fluorescence is quantified using the anisotropy factor, $g_{\text {lum }}$, of CPL, which is given by the equation $g_{\text {lum }}=2\left(I_{\mathrm{L}}-\right.$ $\left.I_{\mathrm{R}}\right) /\left(I_{\mathrm{L}}+I_{\mathrm{R}}\right)$, where $I_{\mathrm{L}}$ and $I_{\mathrm{R}}$ are the intensities of the left- and right-handed circularly polarized emissions, respectively. ${ }^{60,61}$ As shown in Fig. $1 \mathrm{~d}$, in pure THF solution and $f_{\mathrm{w}} 50 \%$, the $g_{\text {lum }}$ values were about $2.1 \times 10^{-4}$ and $2.4 \times 10^{-4}$, respectively. Upon increasing the volume fraction of water, the $g_{\text {lum }}$ value increased by an order of magnitude. The maximum $g_{\text {lum }}$ values for $f_{\mathrm{w}} 85 \%$ and $90 \%$ were $7.2 \times 10^{-3}$ and $2.9 \times 10^{-2}$, respectively. The

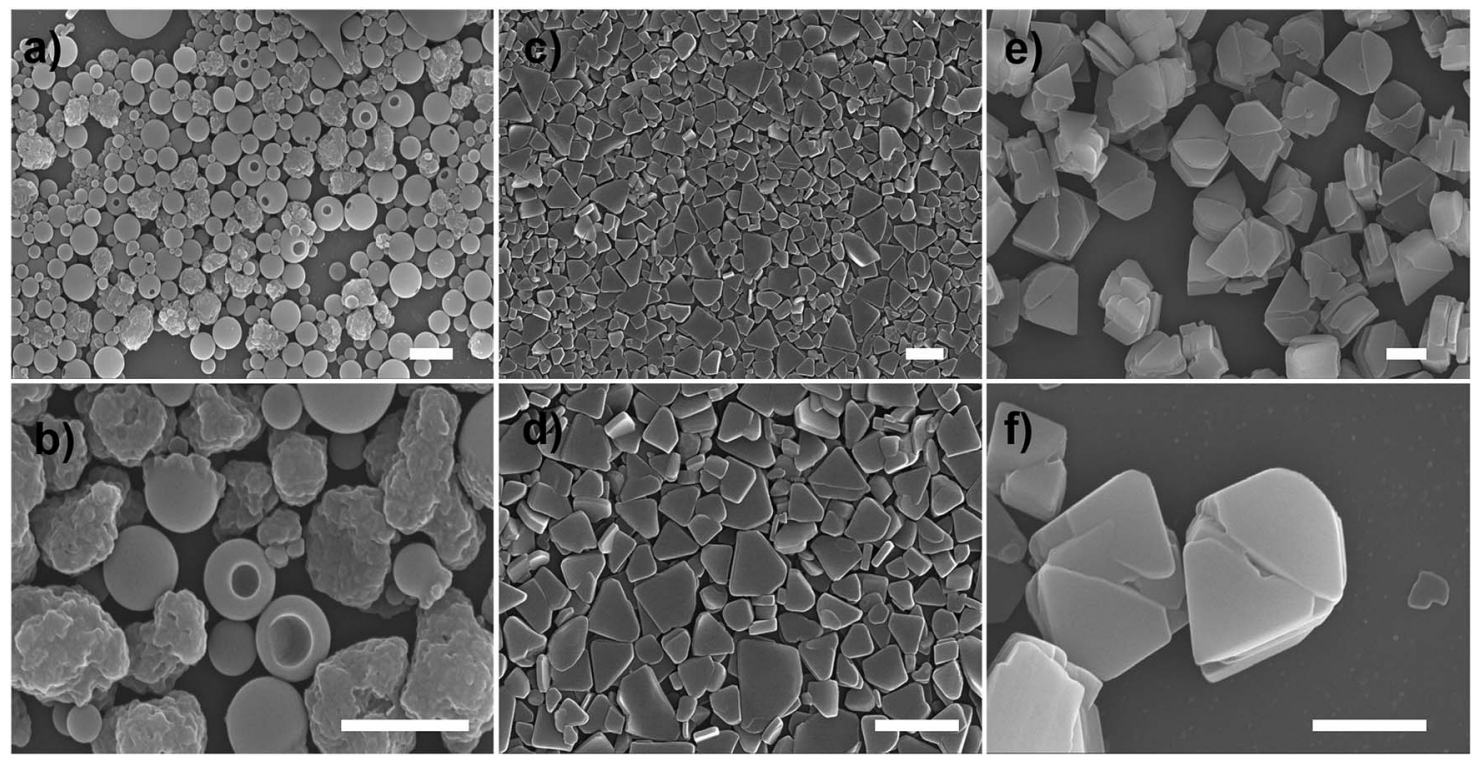

Fig. 2 SEM images of nanostructures of $R$-SPAn in different $f_{\mathrm{w}}$ : (a and b) $50 \%$, (c and d) $85 \%$ and (e and f) $90 \%$, respectively. ([R-SPAn] $=1.5 \mathrm{mM}$, scale bar $1 \mu \mathrm{m}$ ). Upon increasing the fraction of water, the morphologies transformed from $0 \mathrm{D}$ nanospheres with a rough surface or with a hole to 2D and finally to 3D layered nanoflakes. 
a)

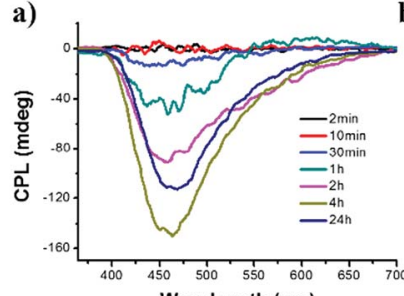

Wavelength $(\mathrm{nm})$

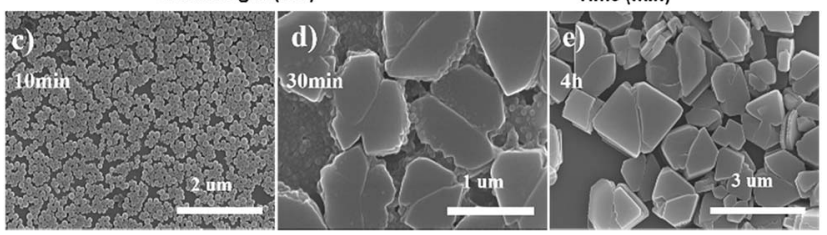

Fig. 3 (a) Aging time-dependent CPL spectra of $R$-SPAn nanostructures in the water fraction of $90 \%$. (b) The plot of $g_{\text {lum }}$ value of nanostructures recorded at different times. Time-dependent SEM images of $R$-SPAn nanostructures in the water fraction of $90 \%$ at (c) $10 \mathrm{~min},(\mathrm{~d}) 30 \mathrm{~min}$ and (e) $4 \mathrm{~h}$, respectively. ([R-SPAn] $=1.5 \mathrm{mM}, \lambda_{\mathrm{ex}}=$ $320 \mathrm{~nm})$

obtained $g_{\text {lum }}$ value for $f_{\mathrm{w}} 90 \%$ in the pure organic systems was

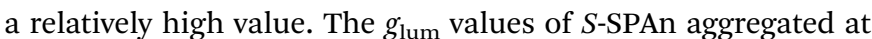
various water fractions are shown in Fig. S6. $\dagger$ The order of

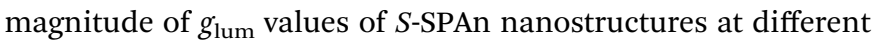
water fractions was the same as that of $R$-SPAn. The obvious amplified $g_{\text {lum }}$ values may have resulted from the strong intermolecular coupling in the nanostructures.

Scanning electron microscopy (SEM) measurement was employed to investigate the morphological transformation of aggregates under different conditions. As shown in Fig. S7a, $\uparrow$ the $R$-SPAn molecule formed amorphous structures upon evaporation of THF. When the water fraction reached $50 \%$, two kinds of nanoparticles with a rough surface and with a hole could be observed (Fig. 2a and b). Upon increasing the fraction of water to $85 \%$, two-dimensional nanoflakes were observed (Fig. 2c and d). Finally, when the water fraction reached 90\%, three-dimensionally stacked flakes, which exhibit the largest $g_{\text {lum }}$ value (Fig. 2e and f), could be obtained. The morphological transformation of $S$-SPAn nanostructures at various water fractions is shown in Fig. S6. $\uparrow$ Thus, by varying the composition of the solvent, a dramatically changed CPL dependent on the morphological transformation could be obtained. We also tried higher volume fractions of water, such as $96 \%$ and $98 \%$. Unlike the sample with a water fraction of $90 \%$, the one with $f_{\mathrm{w}} 96 \%$ exhibited the structure of nanobelts, while hollow microspheres were observed in $f_{\mathrm{w}} 98 \%$ (Fig. S7b and $\mathrm{c}^{\dagger}$ ). However, it is hard to get a plausible CPL signal under these conditions. This was due to the weak luminescence at a higher volume fraction of water. ${ }^{62}$

The ripening process of $R$-SPAn in the water fraction of $90 \%$ was monitored by CPL measurement, as shown in Fig. 3a. The time-dependent CPL investigations in the water fraction of $90 \%$ showed that the CPL intensity dramatically increased. The timedependent emission was also investigated, and it showed a similar tendency to CPL, as shown in Fig. S8. $\dagger$

The $g_{\text {lum }}$ value at different ripening times is shown in Fig. $3 \mathrm{~b}$. In the first two minutes, the $g_{\text {lum }}$ value of the obtained sample a)

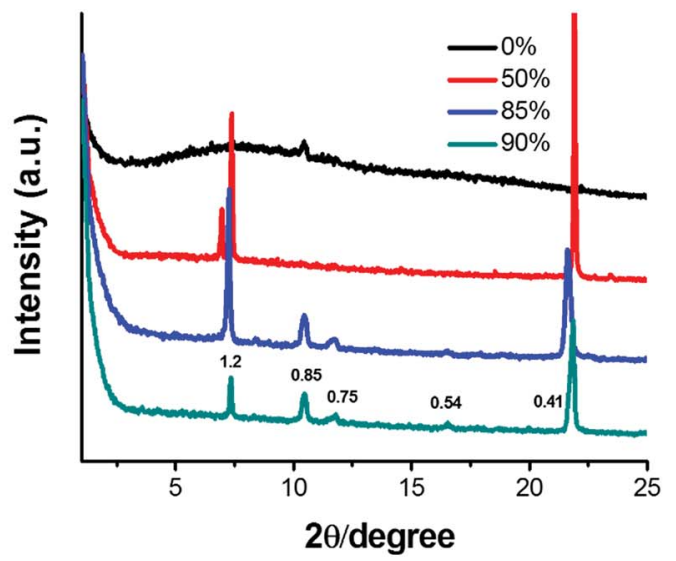

b)

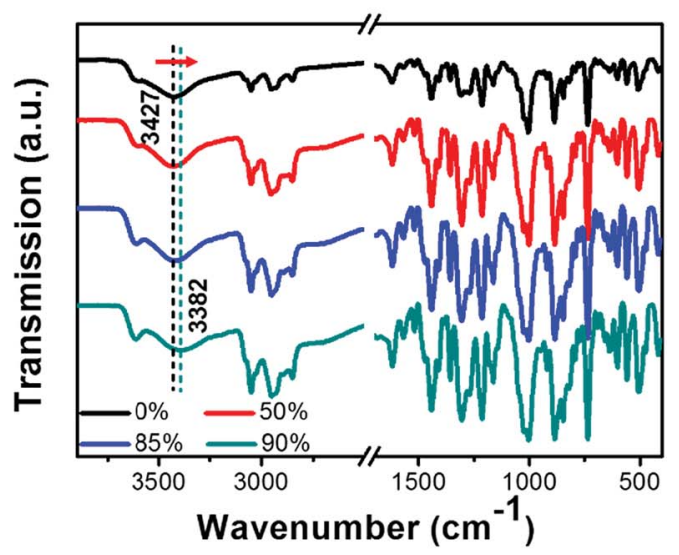

Fig. 4 (a) XRD patterns and (b) FT-IR spectra of self-assembled $R$ SPAn nanostructures formed in various mixed solvents. The drop-cast film of $R$-SPAn solution (THF) was used as $0 \%$ for these tests. [R-SPAn] $=1.5 \mathrm{mM}$. The diffraction peaks of the water fractions of $85 \%$ and $90 \%$ showed a body-centred cubic packing of the $R$-SPAn molecules. Upon increasing the water fraction from $0 \%$ to $90 \%$, the FT-IR peaks ascribed to the stretching vibration of hydroxyl obviously shifted toward lower wavenumbers.

was $2.2 \times 10^{-4}$. After 4 hour ripening, the $g_{\text {lum }}$ value reached $2.6 \times 10^{-2}$, and remained at an almost constant value, confirmed by testing the sample after 24 hours $\left(2.7 \times 10^{-2}\right)$. SEM was carried out to carefully investigate the morphological transformation at different ripening times. In the first 10 minutes, mono-disperse nanoparticles with a size distribution of about $140 \mathrm{~nm}$ were obtained, as shown in Fig. 3c. When the ripening time reached 30 minutes, the nanoparticles stacked together and fused to form a laminated flake structure with rough edges (Fig. 3d). After 4 hours, stacked 3D nanoflakes with a smooth surface were observed (Fig. 3e). Accompanying the morphology transformation from 0D nanospheres to 3D nanoflakes, the CPL activity also showed amplification. The $g_{\text {lum }}$ value was amplified by two orders of magnitude to 0.027 . The enhanced CPL activity of time-dependent morphology transformation in $f_{\mathrm{w}} 90 \%$ suggested that the CPL activity exhibited morphological dependence.

To further clarify the formation of nanostructures with gradually enhanced CPL, X-ray diffraction (XRD) was carried out, as 


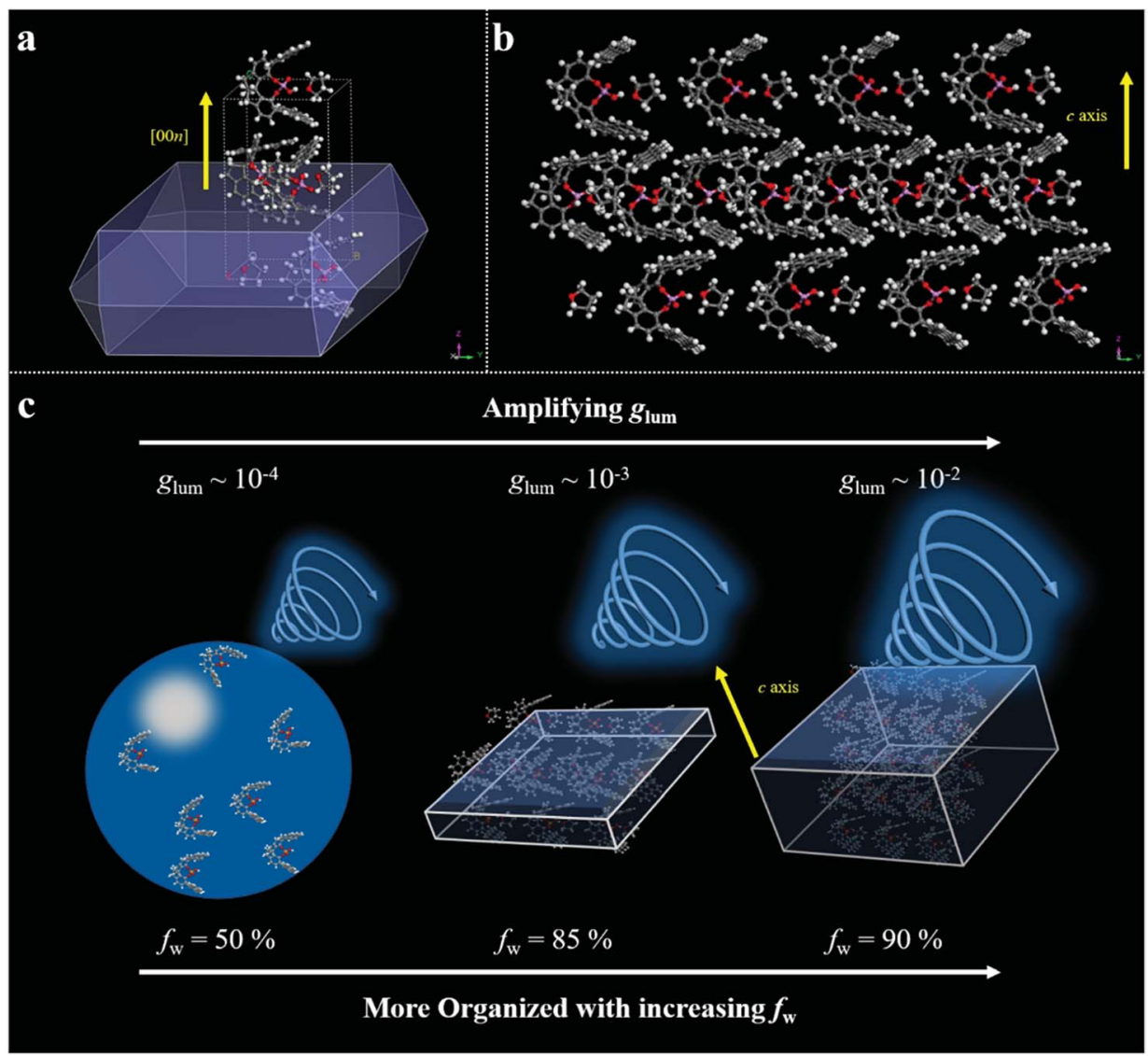

Fig. 5 Molecular packing analysis results and speculated mechanism for the amplification of $g_{\text {lum. }}$ (a) Theoretically predicted growth morphology of an S-SPAn crystal based on the attachment energies calculated with Material Studio package. (b) Crystal packaging along the vertical growth direction. (c) Speculated mechanism for the amplification of $g_{\text {lum. }}$.

shown in Fig. 4a. The diffraction pattern of the $R$-SPAn cast film exhibited only one broad peak which indicated the formation of amorphous structures. For the $f_{\mathrm{w}} 50 \%$ sample, three diffraction peaks were observed at $2 \theta$ values of $7.01^{\circ}, 7.37^{\circ}$ and $21.94^{\circ}$ with $d$ spacings of $1.26 \mathrm{~nm}, 1.19 \mathrm{~nm}$, and $0.41 \mathrm{~nm}$, respectively. Clearly, upon increasing the volume fraction of water to $85 \%$ and $90 \%$, three new diffraction peaks were observed at $2 \theta$ values of $10.46^{\circ}$, $11.71^{\circ}$ and $16.55^{\circ}$. The whole diffraction peaks of $f_{\mathrm{w}} 90 \%$ gave $d$ spacings of $1.20,0.85,0.75$ and $0.54 \mathrm{~nm}$ with a $d$ spacing ratio of about $1: \sqrt{2}: \sqrt{3}: \sqrt{5}$, indicating a body-centred cubic packing of the molecules. ${ }^{63}$ The first order diffraction peak of 3D nanoflakes was located at a $2 \theta$ value of $7.34^{\circ}$ while the peak of $2 \mathrm{D}$ nanoflakes was located at a $2 \theta$ value of $7.24^{\circ}$, which indicated a closer molecular packing of the 3D nanoflakes than the 2D ones. In addition, the selected area electron diffraction (SAED) of the nanostructures obtained at $f_{\mathrm{w}} 85 \%$ and $90 \%$ showed ordered diffraction patterns (Fig. S9 and Table S2 $\dagger$ ). The obtained interlattice spacing was about $0.53-0.54 \mathrm{~nm}$, which could be estimated from the result of XRD. These results suggested that the obtained 2D and 3D nanoflakes had ordered molecular packing and a crystalline nature to some extent.

To gain further insight into the non-covalent interactions, Fourier transform infrared (FT-IR) spectra of the nanostructures were obtained and are shown in Fig. 4b. The peaks located at 885 and $846 \mathrm{~cm}^{-1}$ could be attributed to the $\mathrm{P}-\mathrm{O}$ stretching vibration (aromatic ring). The spectra with sharp peaks at around $1214 \mathrm{~cm}^{-1}$ could be ascribed to the $\mathrm{P}=\mathrm{O}$ stretching vibration. In addition, the peak appearing at $1442 \mathrm{~cm}^{-1}$ could be ascribed to the stretching vibration of $-\mathrm{CH}_{2}$. The band appearing at $3052 \mathrm{~cm}^{-1}$ could be ascribed to the stretching vibration of unsaturated $\mathrm{C}-\mathrm{H}$ single bonds. ${ }^{64}$ Upon increasing the fraction of water, the stretching vibration of the hydroxyl group of the phosphate moiety shifted toward lower wavenumbers, from $3427 \mathrm{~cm}^{-1}$ $\left(f_{\mathrm{w}} 0 \%\right)$ to $3382 \mathrm{~cm}^{-1}\left(f_{\mathrm{w}} 90 \%\right)$. This was indicative of the existence of hydrogen bonding between intermolecular hydroxyl groups. Simultaneously, the peak became broader, which suggested the existence of an enhanced hydrogen bonding. These results indicated that the enhanced intermolecular hydrogen bond might be the main reason for enhancing the molecular stacking of $2 \mathrm{D}$ and 3D nanoflakes with relatively higher $g_{\text {lum }}$ values. ${ }^{65}$

To gain a deep insight into the CPL amplification upon increasing the fraction of water, molecular packing analyses were carried out. We applied Materials Studio as a simulation tool for theoretically predicting the growth morphology of the $S$ SPAn single crystal. The simulation was performed using MS's morphology component based on the attachment energies. Firstly, the single-crystal of S-SPAn was incubated with THF/ water mixed solvent. As shown in Fig. 5a, the crystal morphology of SPAn was plate-like, which was in agreement with the SEM results. The detailed crystallographic data are 
shown in Table S3. $\dagger$ Based on the XRD patterns, the growth in the thickness of the microplates was along the [001] direction, which could be attributed to the molecular packing based on $\pi-$ $\pi$ interaction of anthracene groups and hydrogen bonding between intermolecular hydroxyl groups as shown in Fig. 5b. With all of this in mind, we could speculate on a plausible mechanism for the amplification of CPL dissymmetry factor ( $\left.g_{\text {lum }}\right)$. When the water fraction was relatively low, the SPAn molecules aggregated in the form of microspheres, which was an amorphous packing mode and showed a relatively low $g_{\text {lum }}$ $\left(\sim 10^{-4}\right)$. Upon increasing the water content, SPAn molecules could aggregate in the form of microplates in crystalline packing mode, and the organized packing could amplify the CPL dissymmetry factor $\left(g_{\text {lum }} \sim 10^{-3}\right)$. Furthermore, when the water fraction was elevated to a higher level, more crystal units packed in the thickness direction, i.e., more ordered structures, were involved in the CPL generation process. Thus, $g_{\text {lum }}$ could be further amplified ( $\left.g_{\text {lum }} \sim 10^{-2}\right)$.

\section{Conclusions}

In summary, by tailoring the composition of the solvent (THF/ $\mathrm{H}_{2} \mathrm{O}$ ), the same chiral emitter $R$ - or $S$-SPAn could be constructed into various nanostructures, including $0 \mathrm{D}$ nanospheres, $2 \mathrm{D}$ and 3D nanoflakes. Accompanying the morphological transformation, an amplified circularly polarized emission is observed. The

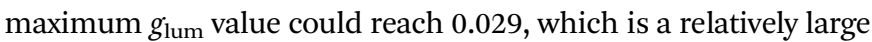
value in pure organic systems. The strong intermolecular interaction resulted in the formation of a more orderly and compact arrangement of nanostructures. The good packing of nanostructures induced a strong excimer emission, which could contribute a large $g_{\text {lum }}$ value. The morphological dependence of emissive nanostructures on CPL activity can lead to controlled modulation of chiroptical properties, offering great potential for fabricating chiroptical organic nanomaterials.

\section{Experimental}

\section{Materials}

(11aS) and (11ab)-3,7-Di-9-anthracenyl-10,11,12,13-tetrahydro5-hydroxy-5-oxide diindeno[7,1de:1',7'-fg][1,3,2] dioxaphosphocin ( $S$-SPAn and $R$-SPAn) were purchased from Daicel Chiral Compounds (Shang Hai) without further purification. Tetrahydrofuran was purchased from TCI. Milli-Q water $(18.2 \mathrm{MO} \mathrm{cm})$ was used in all cases.

\section{Preparation}

$S$-SPAn and $R$-SPAn were dissolved in tetrahydrofuran (THF) with a volume of $1 \mathrm{~mL}, 500 \mu \mathrm{L}, 150 \mu \mathrm{L}$ and $100 \mu \mathrm{L}$, respectively. And then $500 \mu \mathrm{L}, 850 \mu \mathrm{L}$ and $900 \mu \mathrm{L}$ of water were added to the latter three. Thus, the nanostructures with various morphologies could be obtained. The final concentration of $R$ - or $S$-SPAn of all samples was $1.5 \mathrm{mM}$.

\section{Characterization}

UV-vis spectra, fluorescence spectra and CD spectra were obtained using a Hitachi UV-3900, Zolix Omin- $\lambda 500 \mathrm{i}$ monochromator with a photomultiplier tube PMTH-R 928 and JASCO J-810 spectrometers, respectively. CPL measurements were performed with a JASCO CPL-200 spectrometer. XRD analysis was performed on a Rigaku D/Max-2500X-ray diffractometer (Japan) with CuK $\alpha$ radiation $(\lambda=1.5406 \AA)$, operated at a voltage of $40 \mathrm{kV}$ and a current of $200 \mathrm{~mA}$. FTIR studies were performed with a JASCO FTIR-660 spectrometer. SEM was performed on a Hitachi S-4800FE-SEM with an accelerating voltage of $10 \mathrm{kV}$. TEM and selected area electron diffraction were performed on a transmission electron microscope, JEM1011. The fluorescence lifetime measurements were recorded on an Edinburg FLS-980 fluorescence spectrometer using time-correlated single photon counting.

\section{Conflicts of interest}

There are no conflicts to declare.

\section{Acknowledgements}

This work was supported by the National Natural Science Foundation of China (91856115, 51673050, and 21802027); the Ministry of Science and Technology of the People's Republic of China (2017YFA0206600 and 2016YFA0203400); and the Key Research Program of Frontier Sciences, CAS (QYZDJ-SSWSLH044). P. D. is thankful for support from the "New Hundred-Talent Program" research fund of the Chinese Academy of Sciences.

\section{Notes and references}

1 R. A. Hegstrom and D. K. Kondepudi, Sci. Am., 1990, 262, 108-115.

2 J. J. Cornelissen, A. E. Rowan, R. J. Nolte and N. A. Sommerdijk, Chem. Rev., 2001, 101, 4039-4070.

3 G. A. Hembury, V. V. Borovkov and Y. Inoue, Chem. Rev., 2008, 108, 1-73.

4 M. Liu, L. Zhang and T. Wang, Chem. Rev., 2015, 115, 73047397.

5 R. V. Ulijn, J. Mater. Chem., 2006, 16, 2217-2225.

6 A. R. Hirst, S. Roy, M. Arora, A. K. Das, N. Hodson, P. Murray, S. Marshall, N. Javid, J. Sefcik, J. Boekhoven, J. H. van Esch, S. Santabarbara, N. T. Hunt and R. V. Ulijn, Nat. Chem., 2010, 2, 1089-1094.

7 J. Labuta, S. Ishihara, T. Sikorsky, Z. Futera, A. Shundo, L. Hanykova, J. V. Burda, K. Ariga and J. P. Hill, Nat. Commun., 2013, 4, 2188.

8 M. Yamauchi, T. Ohba, T. Karatsu and S. Yagai, Nat. Commun., 2015, 6, 8936.

9 M. Schadt, Annu. Rev. Mater. Sci., 1997, 27, 305-379.

10 C. Wagenknecht, C. M. Li, A. Reingruber, X. H. Bao, A. Goebel, Y. A. Chen, Q. A. Zhang, K. Chen and J. W. Pan, Nat. Photonics, 2010, 4, 549-552. 
11 G. Muller, Dalton Trans., 2009, 44, 9692-9707.

12 M. C. Heffern, L. M. Matosziuk and T. J. Meade, Chem. Rev., 2014, 114, 4496-4539.

13 F. Zinna and L. Di Bari, Chirality, 2015, 27, 1-13.

14 Y. Yang, R. C. da Costa, M. J. Fuchter and A. J. Campbell, Nat. Photonics, 2013, 7, 634-638.

15 I. Sato, R. Sugie, Y. Matsueda, Y. Furumura and K. Soai, Angew. Chem., Int. Ed., 2004, 43, 4490-4492.

16 T. Kawasaki, M. Sato, S. Ishiguro, T. Saito, Y. Morishita, I. Sato, H. Nishino, Y. Inoue and K. Soai, J. Am. Chem. Soc., 2005, 127, 3274-3275.

17 K. Dhbaibi, L. Favereau, M. Srebro-Hooper, M. Jean, N. Vanthuyne, F. Zinna, B. Jamoussi, L. Di Bari, J. Autschbach and J. Crassous, Chem. Sci., 2018, 9, 735-742.

18 N. Saleh, B. Moore IInd, M. Srebro, N. Vanthuyne, L. Toupet, J. A. Williams, C. Roussel, K. K. Deol, G. Muller, J. Autschbach and J. Crassous, Chemistry, 2015, 21, 1673-1681.

19 K. Okano, M. Taguchi, M. Fujiki and T. Yamashita, Angew. Chem., Int. Ed., 2011, 50, 12474-12477.

20 T. Kimoto, N. Tajima, M. Fujiki and Y. Imai, Chem.-Asian J., 2012, 7, 2836-2841.

21 E. M. Sanchez-Carnerero, A. R. Agarrabeitia, F. Moreno, B. L. Maroto, G. Muller, M. J. Ortiz and S. de la Moya, Chemistry, 2015, 21, 13488-13500.

22 F. S. Richardson and J. P. Riehl, Chem. Rev., 1977, 77, 773-792.

23 G. Longhi, E. Castiglioni, J. Koshoubu, G. Mazzeo and S. Abbate, Chirality, 2016, 28, 696-707.

24 J. L. Lunkley, D. Shirotani, K. Yamanari, S. Kaizaki and G. Muller, J. Am. Chem. Soc., 2008, 130, 13814-13815.

25 J. Roose, B. Z. Tang and K. S. Wong, Small, 2016, 12, 64956512.

26 J. Kumar, T. Nakashima and T. Kawai, J. Phys. Chem. Lett., 2015, 6, 3445-3452.

27 E.-M. Roller, L. K. Khorashad, M. Fedoruk, R. Schreiber, A. O. Govorov and T. Liedl, Nano Lett., 2015, 15, 1368-1373.

28 L. He, M. Wang, J. Ge and Y. Yin, Acc. Chem. Res., 2012, 45, 1431-1440.

29 S. Yagai and A. Kitamura, Chem. Soc. Rev., 2008, 37, 15201529.

30 H.-L. Sun, Y. Chen, X. Han and Y. Liu, Angew. Chem., Int. Ed., 2017, 56, 7062-7065.

31 X. Miao, J. Li, B. Zha, K. Miao, M. Dong, J. Wu and W. Deng, Appl. Surf. Sci., 2018, 433, 1075-1082.

32 P. Moitra, Y. Subramanian and S. Bhattacharya, J. Phys. Chem. B, 2017, 121, 815-824.

33 P. Moitra, K. Kumar, P. Kondaiah and S. Bhattacharya, Angew. Chem., Int. Ed., 2014, 53, 1113-1117.

34 H. J. Dou, M. Jiang, H. S. Peng, D. Y. Chen and Y. Hong, Angew. Chem., Int. Ed., 2003, 42, 1516-1519.

35 Y. Li, T. Zhao, C. Wang, Z. Lin, G. Huang, B. D. Sumer and J. Gao, Nat. Commun., 2016, 7, 13777-13788.

36 H. Yao, M. Qi, Y. Liu and W. Tian, Chem.-Eur. J., 2016, 22, 8508-8519.

37 K. V. Rao, D. Miyajima, A. Nihonyanagi and T. Aida, Nat. Chem., 2017, 9, 1133-1139.

38 X. Wang and M. Liu, Chem.-Eur. J., 2014, 20, 10110-10116.
39 H. Maeda, Y. Bando, K. Shimomura, I. Yamada, M. Naito, K. Nobusawa, H. Tsumatori and T. Kawai, J. Am. Chem. Soc., 2011, 133, 9266-9269.

40 E. M. Sanchez-Carnerero, F. Moreno, B. L. Maroto, A. R. Agarrabeitia, M. J. Ortiz, B. G. Vo, G. Muller and S. de la Moya, J. Am. Chem. Soc., 2014, 136, 3346-3349.

41 Z. Shen, T. Wang, L. Shi, Z. Tang and M. Liu, Chem. Sci., 2015, 6, 4267-4272.

42 J. Kumar, H. Tsumatori, J. Yuasa, T. Kawai and T. Nakashima, Angew. Chem., Int. Ed., 2015, 54, 5943-5947.

43 C. Bai and M. Liu, Angew. Chem., Int. Ed., 2013, 52, 2678-2683. 44 B. Han, Acta Phys.-Chim. Sin., 2017, 33, 2325-2326.

45 X. H. Liu, J. M. Jiao, X. X. Jiang, J. F. Li, Y. X. Cheng and C. J. Zhu, J. Mater. Chem. C, 2013, 1, 4713-4719.

46 J. Kumar, T. Nakashima, H. Tsumatori, M. Mori, M. Naito and T. Kawai, Chemistry, 2013, 19, 14090-14097.

47 J. Liu, H. Su, L. Meng, Y. Zhao, C. Deng, J. C. Y. Ng, P. Lu, M. Faisal, J. W. Y. Lam, X. Huang, H. Wu, K. S. Wong and B. Z. Tang, Chem. Sci., 2012, 3, 2737-2747.

48 J. Zhang, Q. Liu, W. Wu, J. Peng, H. Zhang, F. Song, B. He, X. Wang, H. H. Sung, M. Chen, B. S. Li, S. H. Liu, J. W. Y. Lam and B. Z. Tang, ACS Nano, 2019, 13, 3618-3628. 49 D. Yang, P. Duan, L. Zhang and M. Liu, Nat. Commun., 2017, 8, 15727-15736.

50 J. Han, P. Duan, X. Li and M. Liu, J. Am. Chem. Soc., 2017, 139, 9783-9786.

51 X. Yang, J. Han, Y. Wang and P. Duan, Chem. Sci., 2019, 10, 172-178.

52 J. Kumar, T. Nakashima, H. Tsumatori and T. Kawai, J. Phys. Chem. Lett., 2014, 5, 316-321.

53 A. G. Hu, Y. Fu, J. H. Xie, H. Zhou, L. X. Wang and Q. L. Zhou, Angew. Chem., Int. Ed., 2002, 41, 2348-2350.

54 Y. Fu, J. H. Xie, A. G. Hu, H. Zhou, L. X. Wang and Q. L. Zhou, Chem. Commun., 2018, 54, 1449-1452.

55 K. Takaishi, R. Takehana and T. Ema, Chem. Commun., 2018, 54, 1449-1452.

56 M. Inouye, K. Hayashi, Y. Yonenaga, T. Itou, K. Fujimoto, T. Uchida, M. Iwamura and K. Nozaki, Angew. Chem., Int. Ed., 2014, 53, 14392-14396.

57 P. K. Lekha and E. Prasad, Chemistry, 2010, 16, 36993706.

58 Y. Sheng, J. Ma, S. Liu, Y. Wang, C. Zhu and Y. Cheng, Chemistry, 2016, 22, 9519-9522.

59 L. Yang, C.-S. Kwan, L. Zhang, X. Li, Y. Han, K. C.-F. Leung, Y. Yang and Z. Huang, Adv. Funct. Mater., 2018, 18073071807315.

60 J. P. Riehl and F. S. Richardson, Chem. Rev., 1986, 86, 1-16. 61 T. Nakanishi, M. Naito, Y. Takeoka and K. Matsuura, Curr. Opin. Colloid Interface Sci., 2011, 16, 482-490.

62 K. Liu, R. Y. Zhang, Y. X. Li, T. F. Jiao, D. A. Ding and X. H. Yan, Adv. Mater. Interfaces, 2017, 4, 1600183-1600189.

63 I. W. Hamley and V. Castelletto, Prog. Polym. Sci., 2004, 29, 909-948.

64 F. Mangolini, A. Rossi and N. D. Spencer, Tribol. Lett., 2009, 35, 31-43.

65 Y. Sang, P. Duan and M. Liu, Chem. Commun., 2018, 54, 4025-4028. 